\title{
Solution to the Ariadne's thread NMR challenge
}

\section{Reinhard Meusinger}

(C) Springer-Verlag Berlin Heidelberg 2014
The winner of the "Ariadne's thread NMR challenge" (published in volume 406, issue 27) is:

D. Fatta-Kassinos, Dept. of Civil and Environmental Engineering, School of Engineering, University of Cyprus, Nicosia, Cyprus.

The award entitles the winner to select a Springer book of their choice up to a value of $€ 100$.

Our congratulations!

The substance of the Ariadne's thread NMR challenge [1] is linalool (Fig. 1).

For the structural determination we should first consider the ${ }^{13} \mathrm{C}$ NMR spectrum (Fig. 2 in Ref. [1]). Ten carbon signals were detected here. In addition, the DEPT_135 NMR spectrum reveals that two of them are quaternary carbon atoms and three are methylene $\left(\mathrm{CH}_{2}\right)$ carbon atoms. The other carbon atoms are part of two $\mathrm{CH}$ groups and three $\mathrm{CH}_{3}$ groups. With this knowledge, 17 hydrogen atoms are expected for the structure, with an empirical formula $\mathrm{C}_{10} \mathrm{H}_{17}$. This is inconsistent with the 18 hydrogen atoms observed in the ${ }^{1} \mathrm{H}$ NMR spectrum (Fig. 1 in Ref. [1]). Therefore, the hydrogen atom with the broad signal at $2.6 \mathrm{ppm}$ must bond to a heteroatom and the empirical formula becomes $\mathrm{C}_{10} \mathrm{H}_{18} \mathrm{X}$. Without the

This article is the solution to the Analytical Challenge which can be found at http://dx.doi.org/10.1007/s00216-014-8115-y

\section{R. Meusinger $(\bowtie)$}

Institute of Organic Chemistry and Biochemistry,

University of Technology,

Darmstadt, Alarich-Weiss-Str. 4, 64287 Darmstadt, Germany

e-mail: meusi@oc.chemie.tu-darmstadt.de molecular mass given, the determination of this heteroatom is difficult. We can only use the chemical shifts of the carbon atoms. Fortunately an INADEQUATE spectrum was given in this challenge. Here, by means of the chemical shifts (horizontal auxiliary lines) and double quantum coherences (vertical auxiliary lines), the whole carbon skeleton of a compound may be easily traced out. However, beginning with the least shielded carbon signal (1) at $145 \mathrm{ppm}$, the following skeleton will be obtained (Fig. 2).

Here, the carbon atoms are numbered consecutively in descending order of their ${ }^{13} \mathrm{C}$ NMR chemical shifts. Not all connectivities were revealed as clearly as the connectivity of the carbon atoms at chemical-shift-position 5 to the carbon

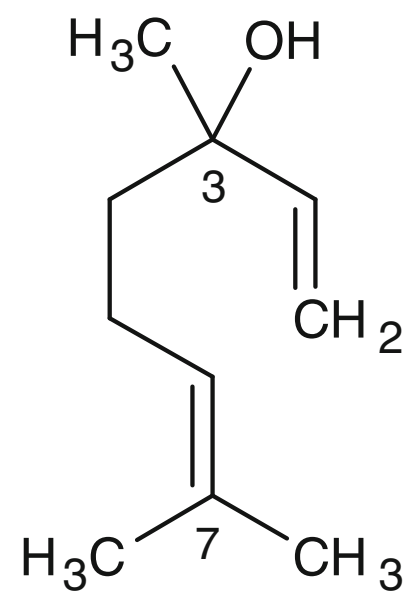

Fig. 1 Linalool $\left(\mathrm{C}_{10} \mathrm{H}_{18} \mathrm{O}, M=154.25 \mathrm{~g} \mathrm{~mol}^{-1}\right)$. IUPAC name: 3,7dimethylocta-1,6-dien-3-ol, other names: $\beta$-linalool, linalyl alcohol, linaloyl oxide, allo-ocimenol 


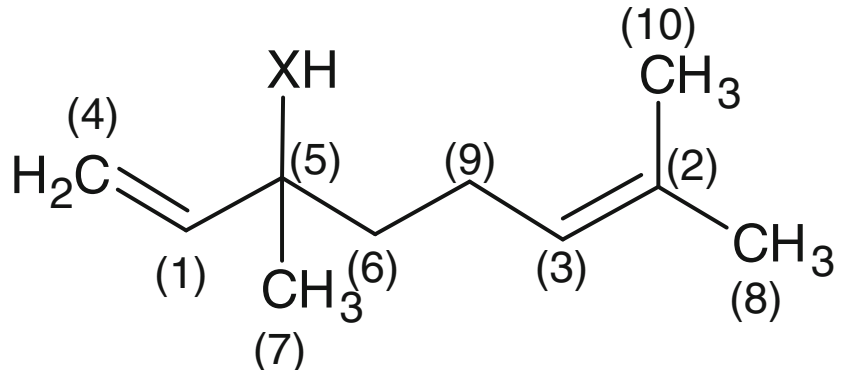

Fig. 2 Working structure of the substance

atoms at positions 1,6 , and 7 . So, the assignment of the lowerintensity carbon signal at shift position 2 to carbon atoms 3 and 10 provides only slight cross peaks in the INADEQUATE spectrum after approximately one day of measuring time.

By consideration of the chemical shift of carbon 5 at $73.5 \mathrm{ppm}$, the heteroatom $\mathrm{X}$ is presumably oxygen. This is in agreement with the weak molecular-ion peak in the mass spectrum at $m / z 154 ;(10 \times 12+18 \times 1+1 \times 16=154)$. Because of this asymmetric quaternary carbon atom, two stereoisomers of linalool exist: the $(R)-(-)$-linalool known as licareol and the $(S)-(+)-$ linalool known as coriandrol. Both isomers are found in nature, for example in lavender and in coriander.

The word coriander comes from the Latin coriandrum which, in turn, originates from the ancient Greek koriannon. This is similar to the name of King Minos' daughter Ariadne, which later evolved to koriannon or koriandron. The synthetically produced linalool analyzed here is a racemic mixture of both isomers. In the year 2000 worldwide linalool production was estimated at 12,000 tonnes. Over half of this amount is believed to be made by chemical synthesis, and the rest is produced from natural plant terpenes [2].

\section{References}

1. Meusinger R (2014) Anal Biol Chem 406:6757-6761

2. Screening Information Dataset (SIDS) (2002) Initial assessment report for SIAM 14. UNEP, Paris 